\title{
Asymptotic estimates for spectral functions connected with hypoelliptic differential operators
}

\author{
By Nils Nilsson
}

\section{Introduction}

Let $x=\left(x_{1}, \ldots, x_{n}\right)$ be coordinates in $R^{n}$ and put $D_{k}=(2 \pi i)^{-1} \partial / \partial x_{k}$ and $D^{\alpha}=D_{1}^{\alpha_{1}} \ldots D_{n}^{\alpha}$. We shall consider a hypoelliptic differential operator $M(D)=\sum M_{\alpha} D^{\alpha}$ with constant coefficients. Let us assume that the coefficients $M_{\alpha}$ are real, so that $M(D)$ is formally self-adjoint. Moreover, we suppose that $M(\xi) \rightarrow+\infty$ when $|\xi| \rightarrow \infty, \xi \in R^{n}$.

If $S$ is an open subset of $R^{n}$ and we define $M(D)$ on $C_{0}^{\infty}(S)$, we get a symmetric linear operator $a_{0}$ in the Hilbert space $L^{2}(S)$. We let $A$ be a self-adjoint extension of $a_{0}$. Then by the spectral theorem $A$ has a spectral resolution $E(\lambda)$ of commuting projection operators increasing with $\lambda$ (Nagy [7]). The operator $E(\lambda)$ is given by a kernel $e_{\lambda}(x, y)$, the spectral function of $A: E(\lambda) u(x)=\int_{S} e_{\lambda}(x, y) u(y) d y$, where $e_{\lambda}$ is infinitely differentiable in $S \times S$. This is proved in Hörmander [5] in the case where $A$ is semi-bounded and in this paper in the general case.

If in particular $S=R^{n}$, there is a unique self-adjoint extension $A_{0}$ of $a_{0}$ with a corresponding spectral function $e_{0, \lambda}$ which is easily computed by a Fourier transformation:

$$
e_{0, \lambda}(x, y)=\int_{M(\xi) \leqslant \lambda} \exp (2 \pi i\langle x-y, \xi\rangle) d \xi
$$

We are going to give a result on the behaviour of $e(\lambda)=e_{0, \lambda}(x, x)$ when $\lambda \rightarrow+\infty$. We shall show (Theorem 1 ) that there are real numbers $a$ and $t, a>0$ and $t$ an integer $\geqslant 0$ such that for some number $k>0$

$$
k^{-1} \lambda^{a}(\log \lambda)^{t} \leqslant e(\lambda) \leqslant k \lambda^{a}(\log \lambda)^{t} \quad(\lambda \text { large })
$$

and

$$
e^{\prime}(\lambda)=0(1) \lambda^{a-1}(\log \lambda)^{t} \quad(\lambda \rightarrow+\infty) .
$$

An analogous result holds for the derivatives of $e_{0, \lambda}$ with respect to $x, y$ :

$$
e_{0, \lambda}^{(\alpha, \alpha)}(x, x)=\int_{M(\xi) \leqslant \lambda} \xi^{2 \alpha} d \xi
$$

If $n=2$, we shall prove a sharper result, namely $e(\lambda)=k(1+o(1)) \lambda^{a}(\log \lambda)^{t}$ for a positive number $k$, and where $t=0$ or $t=1$.

The proof of Theorem 1 uses analytic continuation properties of the function 
$e(\lambda)$, which follow from results in the author's paper [8]. In particular cases the asymptotic behaviour of $e(\lambda)$ has been investigated by Gortjakov [3], who then also computed the numbers $a$ and $t$. Further we prove an asymptotic result for $e_{\lambda}(x, y)$ when $S$ is arbitrary. For this we show an estimate for a fundamental solution of $(M(D)-\lambda)$ when $\lambda \rightarrow-\infty$, and apply a Tauberian theorem of Ganelius for the Stieltjes transformation. We get the following result (Theorem 2), valid also in the not semi-bounded case,

$$
\left|e_{\lambda}(x, x)-e_{0, \lambda}(x, x)\right|=O(1) \lambda^{a-b}(\log \lambda)^{t} \quad(\lambda \rightarrow+\infty),
$$

where $a, t$ correspond to the polynomial $M(\xi)$ as above and $b>0$ is the largest number such that

$$
|\operatorname{grad} M(\xi)| \leqslant C(|M(\xi)|+1)^{1-b} \quad\left(\forall \xi \in R^{n}\right)
$$

for some number $\mathrm{C}$. When $\lambda \rightarrow-\infty$, we have with some $c>0$

$$
e_{\lambda}(x, y)=O(1) \exp \left(-c|\lambda|^{b}\right)
$$

\section{Notations. The spectral function}

We introduce the following customary notations. If $\alpha$ is a multi-index $\left(\alpha_{1}, \ldots, \alpha_{n}\right)$, where the $\alpha_{i}$ are non-negative integers, we put $|\alpha|=\alpha_{1}+\ldots+\alpha_{n}$ and $\xi^{\alpha}=\xi_{1}^{\alpha_{1}} \cdot \ldots \cdot \xi_{n}^{\alpha_{n}}$ with $\xi=\left(\xi_{1}, \ldots, \xi_{n}\right)$. We write $D_{j}=(2 \pi i)^{-1} \partial / \partial x_{j}(j=1, \ldots, n)$ and $D=\left(D_{1}, \ldots, D_{n}\right)$. Let $M(\xi)=\Sigma M_{\alpha} \xi^{\alpha}$ be a complex polynomial in $\xi_{1}, \ldots, \xi_{n}, n \geqslant 2$. Then it corresponds to a differential operator $M(D)=\Sigma M_{\alpha} D^{\alpha}$ with constant coefficients. We shall assume that it is hypoelliptic, i.e. (Hörmander [5]) that

$$
M^{(\alpha)}(\xi) / M(\xi) \rightarrow 0 \quad(|\xi| \rightarrow \infty, \xi \text { real })
$$

where $M^{(\alpha)}(\xi)=D^{\alpha} M(\xi)$, and the relation (1) holds for all $\alpha$ with $|\alpha|>0$. Moreover, we suppose that $M$ is real. Then it follows from (1) that either $M(\xi) \rightarrow+\infty$ or $M(\xi) \rightarrow-\infty$ when $|\xi| \rightarrow \infty(\xi$ real $)$. Let us choose the sign of $M$ so that $M(\xi) \rightarrow+\infty$. Let $S$ be an open subset of $R^{n}$. We shall then work in the Hilbert space $L^{2}(S)$ with inner product $(u, v)=\int_{S} u(x) \overline{v(x)} d x$ and norm $\|u\|=(u, u)^{\frac{1}{2}}$. If we define $M(D)$ on the set $C_{0}^{\infty}(S)$ of all infinitely differentiable functions, which vanish outside compact subsets of $S$, we get a linear operator $a_{0}$ in $L^{2}(S)$, which is also symmetric, since $M$ is real so that $M(D)$ is formally self-adjoint. Let us assume that $A$ is a self-adjoint extension of $a_{0}$ and that $A$ is bounded from below, $A \geqslant \lambda_{0} I$, say, where $I$ is the identity operator in $L^{2}(S)$. In the sense of Nagy [7], to $A$ there corresponds a spectral resolution $E(\lambda)$, which is a projection-valued, non-decreasing function on the real line. We have $E(\lambda)=0$ for $\lambda<\lambda_{0}$. Since $M$ is hypoelliptic, the following statement holds (Hörmander [5]).

To every multi-index $\alpha$ there is a positive integer $r$ such that $D^{\alpha} u$ is continuous (i.e. there is a continuous function $v$ such that $D^{\alpha} u=v$ in the distributional sense) for every distribution $u$ such that $M(D)^{r} u$ is locally square integrable, and we have an inequality

$$
\sup _{x \in K}\left|D^{\alpha} u(x)\right| \leqslant C\left(\left\|M(D)^{r} u\right\|+\|u\|\right)
$$

where $K$ is any compact subset of $S$, and $C$ is independent of $u$ but may depend on 
$\alpha, r, K$, and $S$. Of course, $\sup _{x \in K}\left|D^{\alpha} u(x)\right|$ means $\sup _{x \in K}|v(x)|$, where $v$ is the continuous function equivalent to $D^{\alpha} u$. By (2) it may be shown (Hörmander [5]) that $E(\lambda)$ is given by a kernel $e_{\lambda}(x, y)$, the spectral function of $A$, such that

$$
E(\lambda) u(x)=\int_{S} e_{\lambda}(x, y) u(y) d y \quad\left(u \in L^{2}(S)\right),
$$

where $e_{\lambda}$ is defined and infinitely differentiable in $S \times S$. Further $e_{\lambda}(x, y)=\overline{e_{\lambda}(y, x)}$ for all $x, y \in S$. We also have an estimate

$$
e_{\lambda}^{(\alpha, \beta)}(x, y) \equiv i^{|\alpha+\beta|} D_{x}^{\alpha} D_{y}^{\beta} e_{\lambda}(x, y)=0(1) \lambda^{p+k(|\alpha+\beta|)}
$$

when $\lambda \rightarrow+\infty$, uniformly on compact subsets of $S \times S$, with some positive numbers $p$ and $k$ and all $\alpha, \beta$. For $e_{\lambda}$ we also have the following lemma.

Lemma 1. For any $\alpha$ and any $x \in S, e_{\lambda}^{(\alpha, \alpha)}(x, x)$ is an increasing function of $\lambda$, and the variation with respect to $\lambda$ on any real interval $\Lambda$ satisfies the inequality

$$
\underset{\Lambda}{\operatorname{var}} e_{\lambda}^{(\alpha, \beta)}(x, y) \leqslant\left(\operatorname{var}_{\Lambda} e_{\lambda}^{(\alpha, \alpha)}(x, x) \cdot \operatorname{var}_{\Lambda} e_{\lambda}^{(\beta, \beta)}(y, y)\right)^{\frac{1}{2}}
$$

for all $x, y \in S$ and all $\alpha, \beta$.

Proof. For the proof we refer to Bergendal [1], the Lemmas 1.2.2 and 1.2.1. There the lemma is proved for the spectral function of an elliptic operator, but the proof only uses that $\left(e_{\lambda}-e_{\mu}\right)$ is the kernel of an orthogonal projection if $\lambda>\mu$, and so it works as well in our case.

In particular it follows from the lemma that for any $x, y, \alpha, \beta$ the function $e_{\lambda}^{(\alpha, \beta)}(x, y)$ is locally of bounded variation. If $\lambda<\lambda_{0}$, then $G(\lambda)=(A-\lambda I)^{-1}$ exists as a bounded operator in $L^{2}(S)$, and $\left\|(A-\lambda I)^{-1}\right\| \leqslant\left(\lambda_{0}-\lambda\right)^{-1}$. If the integral of a real function with respect to a spectral measure is defined as in Nagy [7], then $G(\lambda)=\int_{\lambda_{0}}^{+\infty}(\mu-\lambda)^{-1} d E(\mu)$. If in (3) the number $p$ is smaller than 1 , then

$$
G_{\lambda}(x, y)=\int_{\lambda_{0}}^{+\infty}(\mu-\lambda)^{-1} d e_{\mu}(x, y)
$$

is defined as a continuous function in $S \times S$ (this is seen e.g. by an integration by parts). From the definition of the integral with respect to a spectral measure (Nagy [7]) it follows that on $C_{0}^{\infty}(S)$ (and also on $\left.L^{2}(S)\right), G_{\lambda}$ is the kernel of $(A-\lambda)^{-1}$. We shall call $G_{\lambda}$ Green's function corresponding to $A$. We see that for $\varphi \varepsilon C_{0}^{\infty}(S)$ the function $\psi(x)=\left(G_{\lambda}(x, \cdot), \varphi\right)$ is continuous (no correction is needed). If in (3) also $(p+k|\alpha+\beta|)<1$, we get from (3) that $G_{\lambda}^{(\alpha, \beta)}$ is continuous in $S \times S$, and

$$
G_{\lambda}^{(\alpha, \beta)}(x, y)=\int_{\lambda_{0}}^{+\infty}(\mu-\lambda)^{-1} d e_{\mu}^{(\alpha, \beta)}(x, y) .
$$

If instead of $A$ we consider the operator $B=A^{r}$ with a positive integer $r$, then $B$ is self-adjoint and bounded from below, and $B$ is further an extension of $M(D)^{r}$, defined on $C_{0}^{\infty}(S)$. Since $M(D)^{r}$ is hypoelliptic, $B$ has a spectral function $e_{r, \lambda}(x, y)$, and for large $\lambda$ we have $e_{r, \lambda}=e_{\lambda}{ }^{1 / r}$. Hence, taking $r$ large enough, we may make the exponent in (3) smaller than 1, if we have $e_{r, \lambda}$ instead of $e_{\lambda}$. Hence, for any $M, \alpha$ and $\beta,(5)$ holds for the Green's function and the spectral function of $A^{r}$ if we take $r$ large enough. 


\section{N. NILsson, Asymptotic estimates for spectral functions}

We have a particularly simple case when the set $S$ is the whole of $R^{n}$. Then the Fourier transform

$$
\mathcal{I} f(\xi)=\int \exp (-2 \pi i\langle x, \xi\rangle) f(x) d x
$$

taken in the sense of Schwartz [11] is a unitary mapping of $L^{2}(S)$ into $L^{2}\left(R^{n}\right)$, and $\mathfrak{F} a_{0} \mathcal{F}^{-1}$ is multiplication by $M(\xi)$. Hence $a_{0}$ has a unique self-adjoint extension $A_{0}$, and since the spectral resolution $\hat{E}_{r}(\lambda)$ of $\hat{A}_{0}^{r}=\mathcal{F}_{0}^{r} \mathcal{F}^{-1}$ is multiplication by the characteristic function of the set $\left\{\xi \mid M(\xi)^{r} \leqslant \lambda\right\}$ and the operator $\left(\hat{A}^{r}-\lambda\right)^{-1}$ is multiplication by $\left(M(\xi)^{r}-\lambda\right)^{-1}$, we have

$$
e_{0, r, \lambda}(x, y)=\int_{M(\xi)^{r} \leqslant \lambda} \exp (2 \pi i\langle x-y, \xi\rangle) d \xi
$$

and

$$
G_{0, r, \lambda}(x, y)=\int\left(M(\xi)^{r}-\lambda\right)^{-1} \exp (2 \pi i\langle x-y, \xi\rangle) d \xi .
$$

The integral is absolutely convergent for large negative $\lambda$ if $r$ is large enough, since for a hypoelliptic polynomial $M(\xi)$ we have $|M(\xi)| \geqslant C|\xi|^{c}$ for all large real $\xi$ with some positive constants $c$ and $C$ (Hörmander [5]).

We now give a result on the asymptotic behaviour of $e_{0, \lambda}(x, x)$, when $\lambda$ tends to $+\infty$.

Theorem 1. Let $P\left(\xi_{1}, \ldots, \xi_{n}\right)$ be a real polynomial such that $P\left(\xi_{1}, \ldots, \xi_{n}\right) \rightarrow+\infty$ when $|\xi| \rightarrow \infty$ ( $\xi$ real $)$ and let $\alpha$ be a multi-index. If

$$
e(\lambda)=\int_{P(\xi) \leqslant \lambda} \xi^{2 x} d \xi
$$

then there are positive numbers $c, C$, and $a$, and a non-negative integer $t$ such that

and

$$
C^{-1} \lambda^{a}(\log \lambda)^{t} \leqslant e(\lambda) \leqslant C \lambda^{a}(\log \lambda)^{t} \quad(\lambda>c)
$$

If $n=2$, then $t=0$ or $t=1$ and

$$
e(\lambda)=(k+o(1)) \lambda^{\alpha}(\log \lambda)^{t} \quad(\lambda \rightarrow+\infty)
$$

with some positive constant $k$.

Remark. It is clear that the numbers $a$ and $t$ are uniquely determined by $P$ and $\alpha$. We shall call $a=a(P, \alpha)$ and $t=t(P, \alpha)$ the $E$-numbers of the pair $(P, \alpha)$.

The proof of Theorem 1 depends on the following lemma which is a particular case of results in the author's paper [8] (Theorems 1 and 2 and Lemma 2).

Lemma 2. Consider a real algebraic manifold $V(\hat{\lambda}): p(\lambda, \xi)=0$ in $R^{n}$ depending on $\lambda \in R$. Here $p(\lambda, \xi)$ is a real polynomial in $\lambda \in R$ and $\xi \in R^{n}$. Suppose that, for some $\lambda_{0}, V\left(\lambda_{0}\right)$ is not empty and that $\operatorname{grad}_{\xi} p\left(\lambda_{0}, \xi\right) \neq 0$ for all $\xi \in V\left(\lambda_{0}\right)$. Further assume that there is a bounded subset $\Omega$ of $R^{n}$ such that $V(\lambda) \subset \Omega$ for all $\lambda$ in a neighbourhood of $\lambda_{0}$. For $\lambda$ in a neighbourhood of $\lambda_{0}$, let $\omega_{\lambda}(\xi)$ be a differential $(n-1)$-form on $V(\lambda)$ such 
that in any local coordinate system on $V\left(\lambda_{0}\right)$ with coordinates $\xi^{\prime}$ picked among the $\xi_{i}$ (also defining a local coordinate system on $V(\lambda)$ for $\lambda$ in a neighbourhood of $\lambda_{0}$ ) the coefficients of $\omega_{\lambda}(\xi)$ are regular analytic algebraic functions of $\left(\lambda, \xi^{\prime}\right)$. Define the function

$$
g(\lambda)=\int_{V(\lambda)} \omega_{\lambda}(\xi)
$$

in a (sufficiently small) neighbourhood of $\lambda_{0}$. Let $G(\lambda)$ be a primitive function of $g(\lambda)$. Then there is a finite set $W$ of points $\xi_{1}, \ldots, \xi_{r} \in C$ such that $G(\lambda)$ may be continued analytically along any path in $C$ not passing through any point of $W$. Moreover, all the determinations of $G(\lambda)$ in the neighbourhood of any $\lambda \in(C-W)$ span a finite dimensional linear space over C. Put $\varrho=2 \cdot \max _{j}\left|\xi_{j}\right|$. Then, if $\lambda_{1}>\varrho$ and if $G_{1}(\lambda)$ is a function element of $G(\lambda)$ at $\lambda_{1}$, there is a real number $c$ and to every positive integer $N$ a number $K$ such that

$$
|G(\lambda)| \leqslant K|\lambda|^{c}
$$

for all $\lambda$ with $|\lambda|>\varrho$ and for all determinations of $G(\lambda)$ that may be obtained from $G_{1}(\lambda)$ by analytic continuation at most $N$ rounds in the region $|\lambda|>\varrho$. These properties hold also for the function $g(\lambda)$ itself. If $n=2$, then there is a positive integer $N$ such that for $|\lambda|>\varrho$

$$
T^{N} g(\lambda)=g(\lambda)+h(\lambda), \quad T^{N} h(\lambda)=h(\lambda),
$$

where $T$ is analytic continuation one round in the positive sense along circles $|\lambda|=$ constant.

For the proof of Theorem 1 we shall also need the following lemma.

Lemma 3. Let $q\left(\xi_{1}, \ldots, \xi_{n}\right)$ be a complex polynomial. Then there is a number $\sigma$ such that when $|\lambda|>\sigma$ we have $\operatorname{grad} q(\xi) \neq 0$ for all $\xi \in V(\lambda): q(\xi)=\lambda$.

Proof. Consider the algebraic manifold $\operatorname{grad}(q(\xi))=0$. It consists of a finite number of connected components $F_{1}, \ldots, F_{s}$, and $q(\xi)$ is constant $=\lambda_{j}$ on every $F_{j}$. Then for $|\lambda|>\max \left(\left|\lambda_{j}\right|\right)$ we have that grad $q(\xi)$ is different from zero for all $\xi \in V(\lambda)$. The lemma is proved.

Now let us turn to the proof of Theorem 1. It follows from lemma 3 that $e(\lambda)$ is real analytic for $\lambda$ greater than some $\sigma$. Consider the derivative $f(\lambda)=e^{\prime}(\lambda)$. We may write $f(\lambda)$ as an integral over $V(\lambda): P\left(\xi_{1}, \ldots, \xi_{n}\right)=\lambda$,

$$
f(\lambda)=\int_{V(\lambda)} \xi^{2 \alpha}(d \xi / d P(\xi))
$$

It is clear that the differential $(n-1)$-form $\omega_{\lambda}(\xi)=(d \xi / d P(\xi))_{V(\lambda)}$ on $V(\lambda)$ has regular algebraic coefficients in any local coordinate system with coordinates among the $\xi_{i}$. Hence $e(\lambda)$ has the properties stated in Lemma 2 .

From the fact that all the determinations of $e(\lambda)$ span a finite dimensional linear space over the complex numbers it follows (see e.g. Goursat [4], p. 447-460) that in a neighbourhood of infinity $e(\lambda)$ is a finite sum of terms of the type $\lambda^{\beta}(\log \lambda)^{\nu} H(\lambda)$, where $\beta$ is a complex number, $\nu$ a non-negative integer, and $H(\lambda)$ is analytic and singlevalued in a neighbourhood of $\infty$. Hence every such function $H(\lambda)$ may be developed into a Laurent series $\sum_{k=-\infty}^{+\infty} a_{k} \lambda^{k}$, convergent in a neighbourhood of $\infty$. Further all the 
functions $H(\lambda)$ are linear combinations of functions of the form $\lambda^{\gamma}(\log \lambda)^{\mu} h(\lambda)$, where $\gamma$ is a complex number, $\mu$ an integer, and $h(\lambda)$ some branch of $e(\lambda)$. Because of the estimate of $e(\lambda)$ obtained in lemma 2 the Laurent series of any $H(\lambda)$ contains only a finite number of non-zero terms with a positive exponent.

Let us write every term $\lambda^{\beta}(\log \lambda)^{\nu} H(\lambda)$ so that $H(\lambda)=\sum_{k=-\infty}^{0} a_{k} \lambda^{k}$ with $a_{0} \neq 0$, which we can always do, choosing $\beta$ conveniently. Then among the terms of $e$ we select the 'largest' ones, first taking those having $\operatorname{Re}(\beta)$ maximal, $=a$, say, and among these keep those who have $\nu$ maximal, $=t$, say. Then, in every such 'maximal' term we replace $H(\lambda)$ by the constant term in the Laurent expansion. The sum of the selected terms is then a function

$$
\varphi(\lambda)=\lambda^{a}(\log \lambda)^{t}\left(c_{1} \lambda^{i \kappa_{1}}+\ldots+c_{1} \lambda^{i k_{1}}\right)=\lambda^{a}(\log \lambda)^{t} \cdot \Phi(\log \lambda),
$$

where the $c_{i}$ and $k_{i}$ are constants and the $k_{i}$ real, and we may suppose that $\Phi$ is not identically zero. By our method of picking the terms in $\varphi$ we have

$$
e(\lambda)-\varphi(\lambda)=o(1) \lambda^{a}(\log \lambda)^{t} \quad(\lambda \rightarrow+\infty) .
$$

We have $\Phi(\mu)=\Phi_{1}(\mu)+i \Phi_{2}(\mu)$, where $\Phi_{1}$ and $\Phi_{2}$ are functions of the type

$$
g(\mu)=A_{1} \sin \left(d_{1} \mu+e_{1}\right)+\ldots+A_{q} \sin \left(d_{q} \mu+e_{q}\right),
$$

where the $A_{j}, d_{j}$ and $e_{j}$ are real constants and $q$ some positive integer. It is well known (see e.g. Besicovitch [2], p. 5, Th. 12) that a function $g$ of the type (8) has the following property. If $\omega_{0}$ is in the range of $g$, then there is to every $\varepsilon>0$ an increasing sequence $\mu_{1}, \mu_{2}, \ldots$ of real numbers and a positive number $K$, such that $\mu_{j} \rightarrow+\infty$ when $j$ $\rightarrow+\infty, \mu_{j+1}-\mu_{j}<K$ for all $j$ and

$$
\left|g\left(\mu_{j}\right)-\omega_{0}\right|<\varepsilon, j=1,2, \ldots
$$

By this property we get that $\Phi_{2} \equiv 0$. For, if there were a number $y_{0}+0$ in the range of $\Phi_{2}$, then there would be a sequence $\left(\lambda_{j}\right)$, tending to $+\infty$, such that

$$
\left|\operatorname{Im}\left(\varphi\left(\lambda_{j}\right)\right)\right|>\left|y_{0}\right| \lambda_{j}^{a}\left(\log \lambda_{j}\right)^{t} / 2,
$$

and from (7) it would then follow that $e\left(\lambda_{j}\right)$ is non-real, if $j$ is sufficiently large, which is a contradiction, since $e$ is real. Hence $\Phi_{2} \equiv 0$, and $\Phi=\Phi_{1}$. An analogous argument shows that $\Phi \geqslant 0$, as a consequence of the inequality $e(\lambda) \geqslant 0$. Now let us consider $e^{\prime}(\lambda)$.

From the way of picking the terms in $\varphi$ we find

$$
\begin{aligned}
e^{\prime}(\hat{\lambda})=a \lambda^{a-1}(\log \lambda)^{t} \Phi(\log \lambda) & +\lambda^{a-1}(\log \lambda)^{t} \Phi^{\prime}(\log \lambda) \\
& +o(1) \lambda^{a-1}(\log \lambda)^{t} \quad(\lambda \rightarrow+\infty) .
\end{aligned}
$$

By the same type of arguments as above for $\Phi$ and $\Phi_{2}$ we get from (9) and $e^{\prime}(\lambda) \geqslant 0(e(\hat{\lambda})$ is evidently increasing)

$$
\Phi^{\prime}(\mu) \geqslant-a \Phi(\mu) \text {. }
$$

Since $\Phi$ is not identically zero, and $\Phi \geqslant 0$, there is an increasing sequence $\mu_{1}, \mu_{2}, \ldots$ such that $\mu_{j} \rightarrow+\infty$ when $j \rightarrow+\infty$, and with two positive numbers $C$ and $K$

$$
\left(\mu_{j+1}-\mu_{j}\right)<K, \quad \Phi\left(\mu_{j}\right)>C, \text { for all } j .
$$


Let $\mu$ be an arbitrary number $>\mu_{1}$, and let $\mu_{l}$ be the largest $\mu_{j}$ which is $\leqslant \mu$. The solution of the differential equation $u^{\prime}=-a u$ which passes through the point $\left(\mu_{l}, \Phi\left(\mu_{l}\right)\right)$ is

$$
u(x)=\Phi\left(\mu_{l}\right) \exp \left(-a\left(x-\mu_{l}\right)\right) .
$$

From (10) it then follows $\Phi(x) \geqslant u(x)$ for $x \geqslant \mu_{l}$, and so $a$ must be non-negative, since otherwise $\Phi$ would grow exponentially, but we know that it is bounded. Further $x=\mu$ gives

$$
\Phi(\mu) \geqslant C \exp (-a K)>0 .
$$

By (7) we now get the statement about $e(\lambda)$ in the theorem. That about $e^{\prime}(\lambda)$ follows from (9).

It remains to show the stronger assertions in the case $n=2$. By Lemma 2 there is an integer $N>0$ such that in a neighbourhood of $\infty$ we have $T^{N} e^{\prime}=e^{\prime}+h, T^{N} h=h$. Hence $h(\lambda)$ is in a neighbourhood of $\infty$ a single-valued function of $\lambda^{1 / N}$, and so is $F(\lambda)=e^{\prime}(\lambda)-(2 N \pi i)^{-1} h(\lambda) \log \lambda$. Thus $h$ and $F$ may be developed into Puiseux series in a neighbourhood of $\infty$. From the estimate by Lemma 2 holding for $e^{\prime}(\lambda)$ it follows that $h$ and $F$ are of polynomial growth, and so their Puiseux expansions contain only a finite number of non-zero terms with a positive exponent. Hence in a neighbourhood of $\infty$ we have

$$
e^{\prime}(\lambda)=\sum_{k=-\infty}^{k_{0}} a_{k} \lambda^{k / N}+(\log \lambda) \sum_{k=-\infty}^{k_{0} 0^{\prime}} b_{k} \lambda^{k / N}
$$

By integration we find

$$
e(\lambda)=F_{1}(\lambda)+(\log \lambda) F_{2}(\lambda)+b_{-N}(\log \lambda)^{2} / 2,
$$

where $F_{1}$ and $F_{2}$ are Puiseux series, convergent in a neighbourhood of $\infty$ and containing only a finite number of terms with a positive exponent. Since $e(\lambda)$ grows faster than some positive power of $\lambda$, the term $b_{-N}(\log \lambda)^{2} / 2$ is not the leading one, and the particular statement for $n=2$ follows. (It may be shown that actually $b_{-N}=0$.) The theorem is proved.

Now we return to our hypoelliptic polynomial $M(\xi)$ and the unique self-adjoint extension $A_{0}$ in $L^{2}\left(R^{n}\right)$ of $M(D)$, defined on $C_{0}^{\infty}\left(R^{n}\right)$, and the spectral function $e_{0, \lambda}(x, y)=\int_{M(\xi) \leqslant \lambda} \exp (2 \pi i\langle x-y, \xi\rangle) d \xi$. For an arbitrary multi-index $\alpha$ we have

$$
e_{0,1}^{(\alpha, \alpha)}(x, x)=\int_{M(\xi) \leqslant \lambda} \xi^{2 \alpha} d x
$$

Hence Theorem 1 gives a result on the behaviour of $\left.e_{\lambda}^{(\alpha, \alpha)}\right)(x, x)$ when $x \rightarrow+\infty$, and to the pair $(M, \alpha)$ we have a pair of $E$-numbers $a(M, \alpha)$ and $t(M, \alpha)$.

\section{An estimate for a certain fundamental solution}

We consider $\left(M(\xi)^{r}-\lambda\right)$ with a positive integer $r$ and $\lambda$ large and negative. The operator $\left(M(D)^{r}-\lambda\right)$ has a temperate fundamental solution with pole zero which is the inverse Fourier transform of $\left(M(\xi)^{r}-\lambda\right)^{-1}$. Hence the fundamental solution with pole $x$ is

$$
h_{r, \lambda}(x, y)=\int\left(M(\xi)^{r}-\lambda\right)^{-1} \exp (2 \pi i\langle y-x, \xi\rangle) d \xi
$$




\section{N. NILSson, Asymptotic estimates for spectral functions}

where the integral is absolutely convergent if $r$ is large enough. It is clear that $h_{r, \lambda}$ is the complex conjugate of the Green's function of $A_{0}^{r}$ given by (6). We are going to show that outside the pole the fundamental solution tends exponentially to zero, when $\lambda \rightarrow-\infty$. For that we shall need the following lemma.

Lemma 4. If $M(\xi)$ is a hypoelliptic polynomial of degree $m$, then there is a largest number $b=b(M)$ such that $0<b \leqslant 1 / m$ and

$$
\left|M^{(\alpha)}(\xi)\right| \leqslant C(|M(\xi)|+1)^{1-b|\alpha|}
$$

for some number $C$ and all real $\xi$ and all $\alpha$. If $r$ is a positive integer, then $b\left(M^{r}\right)=b(M) / r$.

Proof. For a proof we refer to Hörmander [6], Theorem 3.2, except for the last statement, but this is easily checked using that it is proved in Hörmander [6] that if $b$ is the largest number such that (11) holds for all $\alpha$ with $|\alpha|=1$, then (11) holds for all $\alpha$ with the same $b$.

We also have

Lemma 5. Let $N$ be a hypoelliptic polynomial and put $b=b(\mathrm{~N})$. Then

$$
\left|N^{(\alpha)}\left(\xi+\tau z \xi_{0}\right)-N^{(\alpha)}(\xi)\right| \leqslant C|z|(|\xi|+1)^{-c|\alpha|}\left(|N(\xi)|+\tau^{1 / b}\right)
$$

for some constant $C$, all $\alpha$, all real $\xi$ and $\tau \geqslant 1$ and all complex $z$ with $|z| \leqslant 1$. Here $\xi_{0}$ is arbitrary in $R^{n}$ and $C$ and $c$ are positive and independent of $\xi$, $z$, and $\tau$.

Proof. By Taylor's formula

$$
N^{(\alpha)}\left(\xi+\tau z \xi_{0}\right)-N^{(\alpha)}(\xi)=\sum_{j=1}^{m}(\tau z)^{i} N_{j}(\xi),
$$

where $m$ is the degree of $N$ and $N_{j}$ is a linear combination of derivatives of $N$ of order $(|\alpha|+j)$. By Lemma 4 we have

$$
\left|\tau^{j} z^{j} N_{j}(\xi)\right| \leqslant C \tau^{j}|z|^{j}(|N(\xi)|+1)^{1-b(|\alpha|+j)}
$$

with some constant $C$. From the well-known inequality $x^{a} y^{1-a} \leqslant x+y$ for $x, y>0$ and $0 \leqslant a \leqslant 1$, then, from (12), putting $x=\tau_{:}^{1 / b}, y=(|N(\xi)|+1)$ and $a=j b$, we get

$$
\left|\tau^{j} z^{j} N_{j}(\xi)\right| \leqslant C|z|^{j}(|N(\xi)|+1)^{-b|\alpha|}\left(|N(\xi)|+1+\tau^{1 / b}\right) .
$$

Since $|N(\xi)| \geqslant|\xi|^{k}$ with some positive $k$ for large $|\xi|$ the proof is complete.

From Lemma 4 we also get

Lemma 6. If $N(\xi)$ is hypoelliptic, then

$$
\left|N^{(\alpha)}(\xi)\right| \leqslant C(|\xi|+1)^{-c|\alpha|}(|N(\xi)|+1)
$$

for all $\alpha$ and all real $\xi$, where $c$ and $C$ are positive constants. We may now give an estimate for the fundamental solution considered.

Lemma 7. Let $N(\xi)$ be real and hypoelliptic and let $N(\xi) \rightarrow+\infty$ when $|\xi| \rightarrow \infty$. Put $b=b(N)$. Then 


$$
h_{\lambda}(x, y)=\int \exp (2 \pi i\langle y-x, \xi\rangle)(N(\xi)-\lambda)^{-1} d \xi
$$

is (with respect to $y$ ) a temperate fundamental solution with pole $x$ of $(N(D)-\lambda)$ when $\lambda$ is large and negative, and

$$
D_{y}^{\alpha} h_{\lambda}(x, y)=O(1) \exp \left(-c|\lambda|^{b}\right) \quad(\lambda \rightarrow-\infty)
$$

for $x \neq y$, all $\alpha$ and some $c>0$. The estimate is uniform on compact subsets of the region $x \neq y$.

Proof. Take an arbitrary $\xi_{0} \in R^{n}$, let $z$ be a complex number and put $H_{\lambda}(\xi, z)=$ $N\left(\xi+|\lambda|^{b} z \xi_{0}\right)-\lambda$. By Lemma 5 it follows, taking $\tau=|\lambda|^{b}$, that there are positive numbers $C^{\prime}, C$ and $\left(-\lambda_{1}\right)$ such that

$$
C^{-1}(|N(\xi)|+|\lambda|) \leqslant\left|H_{\lambda}(\xi, z)\right| \leqslant C(|N(\xi)|+|\lambda|) \quad\left(\lambda<\lambda_{1}\right)
$$

for all real $\xi$ and all $z$ with $|z| \leqslant C^{\prime}$.

Now, for $|\operatorname{Im}(z)| \leqslant c^{\prime}$ the inverse Fourier transform of $1 / H_{\lambda}(\xi, z)$ (with respect to $\xi)$ is equal to $\exp \left(2 \pi i z|\lambda|^{b}\left\langle y, \xi_{0}\right\rangle\right) h_{\lambda}(0, y)$. In fact, a translation by $z|\lambda|^{b} \xi_{0}$ corresponds by the Fourier transformation to multiplication by $\exp \left(2 \pi i z|\lambda|^{b}\left\langle y, \xi_{0}\right\rangle\right)$, since $H_{\lambda}(\xi, z)$ keeps away from zero when $|z| \leqslant c^{\prime}$ (see Nilsson [8], p. 114).

Let $B(y)$ be a positive definite homogeneous polynomial of degree $f$. Then $B(y) \exp \left(2 \pi i|\lambda|^{b}\left\langle y, \xi_{0}\right\rangle\right) h_{\lambda}(0, y)$ is (as a function of $\left.y\right)$ the inverse Fourier transform of $B\left(D_{\xi}\right)\left(1 / H_{\lambda}(\xi, z)\right)$. From the rules of differentiation we see that $B\left(D_{\xi}\right)\left(1 / H_{\lambda}(\xi, z)\right)$ is a linear combination of terms $\left(H_{\lambda}^{\left(\alpha_{1}\right)}(\xi, z) \cdot \ldots \cdot H_{\lambda}^{\left(\alpha_{f}\right)}(\xi, z)\right) / H_{\lambda}(\xi, z)^{f+1}$, where $\sum\left|\alpha_{i}\right|=f$.

Now it follows from (13) and Lemma 5 that

$$
\left|H^{\left(\alpha_{i}\right)}(\xi, z) / H_{\lambda}(\xi, z)\right| \leqslant C(|\xi|+1)^{-c\left|\alpha_{i}\right|}
$$

for all real $\xi$, all $\lambda<\lambda_{1}$ and all $z$ with $|z| \leqslant c^{\prime}$, and where $C$ is a constant. So we may conclude that if $f$ is sufficiently large we have

$$
\left|B\left(D_{\xi}\right)\left(1 / \mathrm{H}_{\lambda}(\xi, z)\right)\right| \leqslant C(|\xi|+1)^{-n-1}
$$

with some number $C$, independent of $\xi, \lambda$ and $z$ for $|z| \leqslant c^{\prime}$ and $\lambda<\lambda_{1}$. But then we get, putting $z=i c^{\prime}$ :

$$
\left|B(y) \exp \left(2 \pi c^{\prime}|\lambda|^{b}\left\langle y, \xi_{0}\right\rangle\right) h_{\lambda}(0, y)\right| \leqslant C \quad\left(\lambda<\lambda_{1}\right)
$$

for all $y$, where $C$ is some number, independent of $\lambda$ and $y$.

Since $\xi_{0}$ is arbitrary, the lemma follows in the case $\alpha=0$. To get it for arbitrary $\alpha$ we need only notice that for $y \neq 0$ we have $N\left(D_{y}\right)^{s} h_{\lambda}(0, y)=\lambda^{s} h_{\lambda}(0, y)$ and then use (2).

\section{Asymptotic estimates for the spectral function when the domain $S$ is arbitrary}

First we are going to establish a relation between the Green's functions of $A^{r}$ and $A_{\mathbf{0}}^{r}, G_{r, \lambda}(x, y)$ and $G_{0 r, \lambda}(x, y)=\overline{h_{r, \lambda}(x, y)}$, respectively.

Lemma 8 (see Odhnoff [10]). In $L^{2}(S)$ one has the following identity. 
N. NILsson, Asymptotic estimates for spectral functions

$$
\overline{G_{r, \lambda}(x, \cdot)}=\psi h_{r, \lambda}(x, \cdot)+(B-\lambda)^{-1} k_{r, \lambda}(x, \cdot),
$$

where $x$ is arbitrary in $S, \psi \in C_{0}^{\infty}(S), \psi$ real and $\psi(y)=1$ in a neighbourhood of $x$. Further $B=A^{r}$, and

$$
k_{r, \lambda}(x, y)=\left(\psi(y) B_{y}-B_{y} \psi(y)\right) h_{r, \lambda}(x, y) .
$$

(In particular $k_{r, \lambda}(x, \cdot) \in C_{0}^{\infty}(S)$.)

Proof. Let us denote the right side of (14) by $f_{r, \lambda}(x, \cdot)$ and prove that

$$
\left((B-\lambda) u, f_{r, \lambda}(x, \cdot)\right)=u(x)
$$

when $u \in \mathcal{D}\left(B^{\infty}\right)=\bigcap_{j}^{\infty} \mathcal{D}\left(B^{j}\right) \subset C^{\infty}(S)$ (the last relation by (2)). In fact, we have seen that $\left((B-\lambda) u, \overline{G_{r, \lambda}(x, \cdot)}\right)=u(x)$ for all $u$ such that $(B-\lambda) u \in C_{0}^{\infty}(S)$, and, $(B-\lambda)^{-1}$ being bounded, we should then have $\left(v, f_{r, \lambda}(x, \cdot)-G_{r, \lambda}(x, \cdot)\right)=0$ for all $v \in C_{0}^{\infty}(S)$, and the lemma would follow. To verify (15) we first consider (with $u \in \mathcal{D}\left(B^{\infty}\right)$ )

$$
\begin{aligned}
\left((B-\lambda) u, \psi h_{r, \lambda}(x, \cdot)\right) & =\left(\psi(B-\lambda) u, h_{r, \lambda}(x, \cdot)\right) \\
& =\left((B-\lambda) \psi u, h_{r, \lambda}(x, \cdot)\right)+\left((\psi B-B \psi) u, h_{r, \lambda}(x, \cdot)\right) \\
& =u(x)+\left((\psi B-B \psi) u, h_{r, \lambda}(x, \cdot)\right),
\end{aligned}
$$

where in the last step we have used that $h_{r, \lambda}(x, \cdot)$ is a fundamental solution of $\left(M(D)^{r}-\lambda\right)$ with pole $x$. Now we consider

$$
\begin{aligned}
\left((B-\lambda) u,(B-\hat{\lambda})^{-1} k_{r, \lambda}(x, \cdot)\right) & =\left(u, k_{r, \lambda}(x, \cdot)\right) \\
& =\left(u,(\psi B-B \psi) h_{r, \lambda}(x, \cdot)\right)=\left((B \psi-\psi B) u, h_{r, \lambda}(x, \cdot)\right),
\end{aligned}
$$

where the last step is permitted since the differential operator $(B \psi-\psi B)$ vanishes outside a compact subset of $S-\{x\}$. The lemma now follows from (16) and (17). have

Next we are going to estimate the term $(B-\lambda)^{-1} k_{r, \lambda}(x, \cdot)$ in (14). By Lemma 7 we

$$
\left\|k_{r, \lambda}(x \cdot)\right\|=O(1) \exp \left(-c|\lambda|^{b / r}\right) \quad(\lambda \rightarrow-\infty),
$$

where $c$ is a positive constant and $b$ corresponds to $M$ by Lemma 2 and the estimate is uniform in the neighbourhood of any point in $S$. It follows that

$$
\left\|(B-\lambda)^{-1} k_{r, \lambda}(x, \cdot)\right\|=O(1) \exp \left(-c|\lambda|^{b / r}\right) \quad(\lambda \rightarrow-\infty) .
$$

Let $\alpha$ be an arbitrary multi-index, and let us consider $D^{\alpha}(B-\lambda)^{-1} k_{r, \lambda}(x, \cdot)$. By (2) we then get, if $r$ is large enough,

$$
D_{y}^{\alpha}\left(B_{y}-\lambda\right)^{-1} k_{r, \lambda}(x, y)=O(1) \exp \left(-c^{\prime}|\lambda|^{b / r}\right) \quad(\lambda \rightarrow-\infty)
$$

with a positive constant $c^{\prime}$, and the estimate is uniform on compact subsets of $\omega \times S$, where $\omega$ is a neighbourhood of an arbitrary point in $S$. By Lemma 8 it is then easy to see that

$$
D_{y}^{\alpha}\left(G_{r, \lambda}(x, y)-G_{0, r, \lambda}(x, y)\right)=O(1) \exp \left(-k|\lambda|^{b / r}\right) \quad(\lambda \rightarrow-\infty)
$$

uniformly on compact subsets of $S \times S$, where $k$ is a positive constant. 
Since $\left(G_{r, \lambda}(y, x)-G_{0, r, \lambda}(y, x)\right)=\overline{\left(G_{r, \lambda}(x, y)-G_{0, r, \lambda}(x, y)\right)}$, it also follows that

$$
D_{x}^{\alpha}\left(G_{r, \lambda}(x, y)-G_{0, r, \lambda}(x, y)\right)=O(1) \exp \left(-k|\lambda|^{b / r}\right) \quad(\lambda \rightarrow-\infty)
$$

uniformly on compact subsets of $S \times S$. Hence, with an arbitrary positive integer $s$, if $r$ is large enough

$$
\left(\Delta_{x}^{s}+\Delta_{y}^{s}\right)\left(G_{r, \lambda}(x, y)-G_{0, r, \lambda}(x, y)\right)=O(1) \exp \left(-k|\lambda|^{b / r}\right) \quad(\lambda \rightarrow-\infty)
$$

uniformly on compact subsets of $S \times S$. By well-known estimates for elliptic operators (of the type (2)) it then follows that for any pair $(\alpha, \beta)$ of multi-indices

$$
\left(G_{r, \lambda}^{(\alpha, \beta)}(x, y)-G_{0, r, \lambda}^{(\alpha, \beta)}(x, y)\right)=O(1) \exp \left(-k|\lambda|^{b / r}\right) \quad(\lambda \rightarrow-\infty),
$$

if $r$ is large enough.

If we assume $A>0, A_{0}>0$, we have by (6) and (18)

$$
\int_{0}^{+\infty}(\mu-\lambda)^{-1} d\left(e_{r, \mu}^{(\alpha, \beta)}(x, y)-e_{0, r, \mu}^{(\alpha, \beta)}(x, y)\right)=O(1) \exp \left(-k|\lambda|^{b / r}\right)
$$

when $\lambda \rightarrow-\infty$. To get information for $\left(e_{r, \lambda}-e_{0, r, \lambda}\right)$ from this estimate we shall use a Tauberian theorem by Ganelius. The theorem to be quoted is unpublished but will appear in the Mathematica Scandinavica; the corresponding theorem for the La. place transformation has been announced in [12]. (If we are content with the result $e_{\lambda}(x, x)=(1+o(1)) e_{0, \lambda}(x, x)$ we can use a Tauberian theorem by Keldish [13], where the Tauberian condition is

$$
O \leqslant\left(\frac{\partial}{\partial \lambda} e_{r, \lambda}(x, x)\right) / e_{r, \lambda}(x, x) \leqslant 1 .
$$

It follows from Theorem 1 that this condition is satisfied, if $r$ is large enough.)

First we define a slowly oscillating function as a positive, continuous function $L$ on the positive real line such that $L(c \omega) / L(\omega) \rightarrow 1$ when $\omega \rightarrow+\infty$ for every $c>0$. Then we have

Lemma 9. Let the function $\sigma(\mu)$ be locally of bounded variation for $\mu>0$. Suppose that $\int_{0}^{+\infty}(\mu+\omega)^{-1} d \sigma(\mu)$ is convergent for $\omega=$ some $x_{0}>0$. (and hence for every $\omega$ not on the negative real axis). Let $c, x$, and $\nu$ be real numbers, $c>0,0<x \leqslant \frac{1}{2}$ and $\nu<1$. Let $L(\omega)$ be a slowly oscillating function. Then, if

$$
\int_{0}^{-\infty}(\mu+\omega)^{-1} d \sigma(\mu)=O(1) \exp \left(-c|\omega|^{\alpha}\right) \quad(\omega \rightarrow+\infty)
$$

and

$$
\sup \left(\int_{\substack{\omega \\ \omega \leqslant \Omega \leqslant \omega+\omega^{1-\varkappa}}}^{\Omega} d \sigma(\mu)\right) \leqslant O(1) \omega^{\nu-\varkappa} L(\omega) \quad(\omega \rightarrow+\infty)
$$

then

$$
\sigma(\omega)=O(1) \omega^{\nu-x} L(\omega) \quad(\omega \rightarrow+\infty) .
$$

Now we are going to apply this Tauberian theorem to the function

$$
\sigma(\mu)=\left(e_{0, r, \mu}^{(\alpha, \alpha)}(x, x)-e_{r, \mu}^{(\alpha, \alpha)}(x, x)\right)
$$




\section{N. NILSson, Asymptotic estimates for spectral functions}

with $\alpha$ arbitrary and $x \in S$. We let $(a, t)$ be the $E$-numbers of $(M, \alpha)$. With $c=$ the number $k$ of $(18), \varkappa=b / r, v=a / r$ and $L(\omega)=(\log \omega)^{t}$ we have that $c, x, v$, and $L$ satisfy the conditions of the lemma, if $r$ is large enough, and also the other conditions are satisfied, (19) because of (18) and (20) because of the estimate for $(\partial / \partial \lambda) e_{0, r, \lambda}^{(\alpha, \alpha)}(x, x)$ in Theorem 1 and the fact that $e_{r, \lambda}^{(\alpha, \alpha)}(x, x)$ is a non-decreasing function of $\lambda$, which was stated in Lemma 1. Hence we get the conclusion of Lemma 9:

$$
\left(e_{0, r, \lambda}^{(\alpha, \alpha)}(x, x)-e_{r, \lambda}^{(\alpha, \alpha)}(x, x)\right)=O(1) \lambda^{(a-b) / r}(\log \lambda)^{t} \quad(\lambda \rightarrow+\infty) .
$$

By (21) and Lemma 1 we now get

$$
\operatorname{var}_{\left(\lambda, \lambda+\lambda^{1-b / r)}\right.} e_{r, \lambda}^{(\alpha, \beta)}(x, y)=O(1) \lambda^{\left(a_{\alpha}+a_{\beta}-2 b\right) / 2 r}(\log \lambda)^{\left(t_{\alpha}+t_{\beta}\right) / 2}
$$

when $\lambda \rightarrow+\infty$. Here $\left(a_{2}, t_{1}\right)$ and $\left(a_{2}, t_{2}\right)$ are the $E$-numbers of $(M, \alpha)$ and $(M, \beta)$, respectively, and $\alpha$ and $\beta$ are arbitrary multi-indices, $x$ and $y$ belong to $S$, and $r$ is sufficiently large. The same estimate holds for $e_{0}^{(\alpha, \alpha)}(x, y)$, and so, taking

$$
\sigma(\mu)=\left(e_{0, r, \mu}^{(\alpha, \beta)}(x, y)-e_{r, \mu}^{(\alpha, \beta)}(x, y)\right),
$$

we get by the Tauberian theorem

$$
\left(e_{0, r, \lambda}^{(\alpha, \beta)}(x, y)-e_{r, \lambda}^{(\alpha, \beta)}(x, y)\right)=O(1) \lambda^{\left(a_{1}+a_{2}-2 b\right) / 2 r}(\log \lambda)^{\left(t_{1}+t_{2}\right) / 2}
$$

when $\lambda \rightarrow+\infty$. However, we want the results for $e_{\lambda}=e_{1, \lambda}$ and not for $e_{r, \lambda}$. From the relation $e_{r, \lambda}=e_{\lambda^{1 / r}}$ we immediately find that $(22)$ is valid not only for $r$ sufficiently large but also for $r=1$. Our restriction that $A>0, A_{0}>0$, may also beremoved, since by a translation in the eigenvalue parameter $\lambda$ we may make these two inequalities satisfied, and the translation does not change the asymptotic formulas.

We can also take care of the case where $A$ is not bounded from below. We have the following lemma.

Lemma 10. Let $A$ be an arbitrary self-adjoint extension in $L^{2}(S)$ of $a_{0}$ and $E(\lambda)$ the corresponding spectral resolution. Then for any $\lambda, E(\lambda)$ is given by a kernel $e_{\lambda}$ :

$$
E(\lambda) u(x)=\int_{S} e_{\lambda}(x, y) u(y) d y \quad\left(u \in L^{2}(S)\right)
$$

where $e_{\lambda}$ is infinitely differentiable in $S \times S$ and where

$$
e_{\lambda}^{(\alpha, \beta)}(x, y)=O(1) \exp \left(-c|\lambda|^{b(M)}\right) \quad(\lambda \rightarrow-\infty)
$$

uniformly on compact subsets of $S \times S$. Here $c$ is a positive constant and $\alpha, \beta$ are arbitrary multi-indices.

Proof. For a proof we refer to Nilsson [8], the Theorems 3 and 4, where the corresponding theorem is proved for an elliptic differential operator $P(D)$. The proof, however, works as well in our case. For it uses essentially three facts:

(a) To every $x \in S$ we have a fundamental solution $g_{\lambda}(x, y)$ with pole $x$ of $(P(D)-\lambda)$, defined when $\lambda$ is large and negative and decreasing exponentially outside the pole when $\lambda \rightarrow-\infty$, 
(b) the fundamental solution $g_{\lambda}(x, y)$ above satisfies an inequality

$$
\left|g_{\lambda}(x, y)\right| \leqslant C \cdot|x-y|^{-n+\delta} \quad(x \neq y)
$$

uniformly on compact subsets of $S \times S$ and for all $\lambda$. Here $C$ and $\delta$ are positive constants,

(c) we have for $P(D)$ an interior a priori $L^{2}$-estimate of the type (2) (this paper).

Now $(a)$ holds also for $M(D)$ (Lemma 7 ) and so does $(c)$. Further in [8] (b) is only used to make certain that the mapping

$$
u \rightarrow \int_{K} g_{\lambda}(\cdot, y) u(y) d y \quad(K=\operatorname{compact} \subset S)
$$

is continuous from local $L^{2}$ to local $L^{2}$ and that the continuity is uniform with respect to $\lambda$. In our case we have that $M(\xi) \geqslant C|\xi|^{k}$ when $\xi$ is large, with some positive $c$, $C$, and it follows that the temperate fundamental solution of $\left(M(D)^{r}-\lambda\right)$ is uniformly bounded with respect to $\lambda$, if $r$ is large enough and $\lambda$ large and negative.

This result may then replace $(b)$ in question of $M(D)^{r}$, but via the elementary connection between spectral functions of $M(D)$ and $M(D)^{r}$, with $r$ odd, we get the desired result also for $M(D)$.

By Lemma 10 we may now see that (22) is valid also if $A$ is not bounded from below. For let us consider $A^{r}$ with $r$ even; then $A^{r}$ is bounded from below so that (22) holds for $e_{r, \lambda}$. But $e_{\lambda}=e_{-\lambda}+e_{r, \lambda r}$ for $\lambda>0$, and so by lemma 10 we get (22) also for $e_{\lambda}$.

We collect our results in the following theorem.

Theorem 2. Let $M(\xi)$ be a real hypoelliptic polynomial in $R^{n}, n \geqslant 2$, such that $M(\xi) \rightarrow+\infty$ when $|\xi| \rightarrow \infty$. Let $S$ be an open subset of $R^{n}$ and let $a_{0}$ be the operator in $L^{2}(S)$ defined by the differential operator $M(D)$, acting on $C_{0}^{\infty}(S)$. Suppose that $A$ is a self-adjoint extension in $L^{2}(S)$ of $a_{0}$, not necessarily bounded from below. Then the spectral resolution $E(\lambda)$ of $A$ is given by a kernel $e_{\lambda}(x, y)$ :

$$
E(\lambda) u(x)=\int_{S} e_{\lambda}(x, y) u(y) d y \quad\left(u \in L^{2}(S)\right)
$$

where $e_{\lambda}$ is infinitely differentiable in $S \times S$, and

$$
e_{\lambda}^{(\alpha, \beta)}(x, y)=O(1) \exp \left(-c|\lambda|^{b(M)}\right) \quad(\lambda \rightarrow-\infty)
$$

for any multi-indices $\alpha, \beta$. Here $c>0$, and $b(M)$ is the largest positive number $b$ such that with some constant $C$

$$
\left|M^{(\alpha)}(\xi)\right| \leqslant C(|M(\xi)|+1)^{1-b|\alpha|}
$$

for all $\alpha$ and all real $\xi$. (If $M$ is elliptic and of degree $m$, we have $b(M)=1 / m$ ). Further, if $A_{0}$ is the unique self-adjoint extension in $L^{2}\left(R^{n}\right)$ of $M(D)$, defined on $C_{0}^{\infty}\left(R^{n}\right)$, and $e_{0, \lambda}$ its spectral function, then

$$
\left(e_{\lambda}^{(\alpha, \beta)}(x, y)-e_{0, \lambda}^{(\alpha, \beta)}(x, y)\right)=O(1) \lambda^{\left(t \alpha^{+t_{\beta}}-2 b(M)\right)}(\log \lambda)^{\left(t_{\alpha^{+}}+t_{\beta}\right) / 2}
$$




\section{N. NILSSON, Asymptotic estimates for spectral functions}

when $\lambda \rightarrow+\infty$. Here $\alpha, \beta$ are arbitrary. The pair $\left(a_{\gamma}, t_{\gamma}\right)$ is characterized by the property that

$$
K^{-1} \lambda^{a} \gamma(\log \lambda)^{t} \alpha \leqslant e_{0, \lambda}^{(\gamma, \gamma)}(x, x) \leqslant K \lambda^{a_{\gamma}}(\log \lambda)^{t_{\gamma}}
$$

for some $K>0$, all large positive $\lambda$ and $\gamma=\alpha, \beta$. That such numbers exist was proved in Theorem 1 .

\section{B I B L I O G R A P H Y}

1. Bergendat, G., Convergence and summability of eigenfunction expansions connected with ellipitic differential operators. Medd. fr. Lunds univ. mat. sem 15 (1959).

2. Besicovitch, A. S., Almost Periodic Functions. Cambridge, 1932.

3. Ganelius, J., Un théorème taubérien pour la transformation de Laplace. C. R. Acad. Sci. Paris 242, 719-721 (1956).

4. GorTJakov, V. N., On the asymptotic behaviour of the spectral function of a class of hypoelliptic operators. Doklady Ak. Nauk, 152,3, p. 519-522 (1963).

5. Goursat, E., Cours d'analyse mathématique 2. Paris, 1924.

6. Hörmander, L., On the theory of general partial differential operators. Acta Mathematica $94(1955)$.

7. - On interior regularity of the solutions of partial differential equations. Comm. of pure and appl. math. 9 (1958).

8. Keldish, M. V., On a Tauberian theorem. Trudy Matematitjeskovo Instituta Imeni V. A. Steklova, vol. XXXVIII, 77-86 (1951).

9. Sz-NAGY, B., Spektraldarstellung linearer Transformationen des Hilbertsehen Raumes. Berlin, 1942.

10. NiLsson, N., Some estimates for eigenfunction expansions and spectral functions corresponding to elliptic differential operators. Math. Scand. 9 (1961).

11. - - Some growth and ramification properties of certain integrals on algebraic manifolds. Arkiv för matematik 5,463 (1964).

12. Ophnoff, J., Operators generated by differential problems with eigenvalue parameter in equation and boundary condition. Medd. fr. Lunds univ. mat. sem. 14 (1959).

13. Schwartz, L., Théorie des distributions, I-II. Paris, 1950-1951. 